\title{
Static and dynamic wetting of soft substrates
}

\author{
Longquan Chen a,b,*, Elmar Bonaccurso ${ }^{\mathrm{c}, * *}$, Tatiana Gambaryan-Roisman ${ }^{\mathrm{d}}$, Victor Starov ${ }^{\mathrm{e}}$, \\ Nektaria Koursari ${ }^{\mathrm{e}}$, Yapu Zhao ${ }^{\mathrm{f}, \mathrm{g}}$ \\ a Institute of Fundamental and Frontier Sciences, University of Electronic Science and Technology of China, Chengdu 610054, PR China \\ b School of Mechanics and Engineering, Southwest Jiaotong University, Chengdu 610031, PR China \\ ' AIRBUS Central R \& T, Materials X, 81663 Munich, Germany \\ d Institute for Technical Thermodynamics and Center of Smart Interfaces, Technische Universität Darmstadt, Alarich-Weiss-Str. 10, 64287 Darmstadt, Germany \\ e Department of Chemical Engineering, Loughborough University, Loughborough LE11 3TU, UK \\ f State Key Laboratory of Nonlinear Mechanics, Institute of Mechanics, Chinese Academy of Sciences, Beijing 100190, PR China \\ ${ }^{g}$ School of Engineering Science, University of Chinese Academy of Sciences, Beijing 100049, PR China
}

\section{Current Opinion in Colloid \& Interface}

Science (2018) 35, 46-57

This review comes from a themed issue on

Wetting and Spreading

Editors: Victor M. Starov and Hans-Jürgen Butt

For a complete overview see the Issue and

the Editorial

\section{Article History:}

Received 17 November 2017

Received in revised form 4 December 2017

Accepted 6 December 2017

Available online 20 December 2017

https://doi.org/10.1016/j.cocis.2017.12.001

1359-0294/@ 2017 Elsevier Ltd. All rights

reserved.

Keywords:

Capillary force

Disjoining pressure

Elastocapillary length

Substrate deformation

Contact angle

Contact angle hysteresis

Dynamic wetting

Capillary phenomena

\begin{abstract}
A B S T R A C T
A survey of recent literature on wetting phenomena reveals that there is a fast-growing interest in wetting of soft or deformable substrates, due to its potential applications in many industrial, technical and biological processes. Unlike rigid substrates, a droplet deposited on a soft substrate deforms the substrate via a combination of the normal component of surface tension and the Laplace pressure, i.e. by capillary force and the action of disjoining pressure. In turn, the capillary and disjoining pressure-induced substrate deformation affects the wetting phenomena on the substrate. In this review, we summarize recent achievements on static and dynamic wetting of soft substrates and provide an outlook to future progress. In static wetting, theoretical, numerical and experimental investigations of capillary and disjoining pressure-induced substrate deformation are introduced, and corresponding effects on contact angle and contact angle hysteresis are discussed. In dynamic wetting, the influence of substrate stiffness on spontaneous wetting, droplet impact dynamics, and other types of forced wetting and dewetting is considered. Finally, other interesting capillarity-controlled phenomena occurring on soft and softlike substrates are briefly introduced.
\end{abstract}

(C) 2017 Elsevier Ltd. All rights reserved.

\section{Introduction}

The wetting of solid substrates by liquids is crucial for a variety of practical applications such as spray coating, surface cooling, oil recovery, pesticide deposition and inkjet printing [1-3]. When a droplet is brought into contact with a solid substrate, it spreads out spontaneously in the case of complete or partial wetting to minimize the total free energy of the system. Under the assumption that the solid substrate is

\footnotetext{
* Correspondence to: L. Chen, Institute of Fundamental and Frontier Sciences, University of Electronic Science and Technology of China, Chengdu 610054, PR China.

** Corresponding author.

E-mail addresses: lqchen@uestc.edu.cn lqchen@swjtu.edu.cn (L. Chen), elmar.bonaccurso@airbus.com (E. Bonaccurso).
}

rigid, smooth and chemically isotropic, Thomas Young first derived the equation for the contact angle of the droplet on the substrate at equilibrium, i.e. the well-known Young's equation [4]: $\gamma \cos \theta_{e q}=\gamma_{S V}$ $-\gamma_{S L}$, where $\theta_{e q}$ is the Young contact angle; $\gamma, \gamma_{S V}, \gamma_{S L}$ are the liquidvapor, solid-vapor and solid-liquid interfacial tensions, respectively. Natural substrates are frequently decorated with a variety of physical or chemical moieties. For such heterogeneous substrates the Wenzel [5] or Cassie [6] equations, which are modifications of the Young's equation, are widely employed to calculate the contact angle. The above three equations have long been recognized as the useful relations for surface engineers to design and control the wettability of solid substrates [1,7-9].

Although Young's equation is useful to explain wetting phenomena in nature, it only accounts for the in-plane balance of the three 
(a)

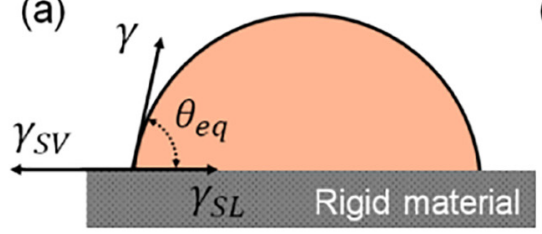

(b)

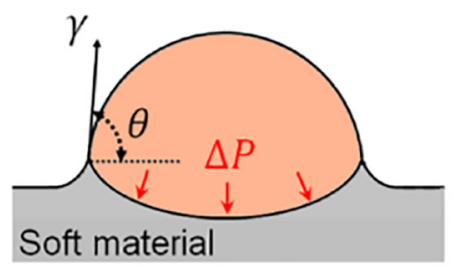

Fig. 1. Sketch of a liquid droplet deposited on a rigid (a) and on a soft (b) substrate. The soft substrate is deformed by surface tension and Laplace pressure $\Delta P$ of the droplet.

interfacial tensions near the three-phase contact line (TPCL - Fig. 1a). The vertical component of the liquid surface tension, $\gamma \sin \theta_{e q}$, remains unbalanced and results in a vertical net force applied on the solid substrate at the TPCL (Fig. 1b). Moreover, the Laplace pressure [1], which is inversely proportional to the droplet curvature $R_{0}$, i.e. $\Delta P \propto \gamma / R_{0}$, is exerted to the liquid-solid interface (Fig. 1b). Consequently, a socalled "wetting ridge", $\delta$, with a length scale of the order of the elastocapillary length, $\delta \sim L_{e c}=\gamma / G$, is formed near the TPCL $[10,11]$, where $G$ is the elastic/shear modulus of the substrate material. For rigid solids like silicon and steel $(G \sim 100 \mathrm{GPa}), \delta$ is of molecular scale and its influence on the contact angle can safely be ignored. In contrast, for soft solids such as biological tissues and polymer gels ( $G \sim 1 \mathrm{kPa}), \delta$ can be up to sub-millimetre scale and thus it can strongly affect the wettability. In the static case of a sessile droplet deposited on a soft substrate, the substrate deformation leads to a rebalance of the interfacial tensions and to a modification of the contact angle [12"] and of the contact angle hysteresis [13]. In the dynamic case of a droplet spreading on a soft substrate, the movement of the wetting ridge with the contact line causes additional energy dissipation and thus influences dynamic wetting $\left[14^{*}, 15,16\right]$, adhesion [17,18], frost growth [19], drop evaporation $[20,21]$ and condensation [22], just to name a few processes.

Since the pioneering works of Lester [10], Rusanov $\left[23^{*}, 24\right]$, Deryagin et al. [25], Shanahan and de Gennes [11,26], wetting on soft substrates has attracted the attention of scientists and engineers, but the recognition of its importance has not become completely understood until recently. The recent progress could be due to the development of experimental microscopic imaging techniques that allow detecting interfacial droplet profiles and substrate deformations at high spatial/temporal resolution and new theoretical and experimental work performed over the past decade $[27,28]$. Below an overview on the latest progress on this topic is attempted. In Section 2, the theoretical, numerical and experimental investigations on substrate deformation are summarized. In addition, it is discussed how the deformation influences the wetting properties of soft substrates, i.e., the contact angle and contact angle hysteresis. Section 3 is devoted to dynamic wetting of soft substrates, where the effects of substrate deformation on spontaneous and forced wetting are presented. In Section 4, further capillary phenomena on soft substrates, thin films, cantilevers, flexible fibres, and liquid-infused substrates are introduced. Finally, the latest achievements in "soft wetting" are briefly summarized and an outlook is given, which, according to the authors' opinion, could stimulate further investigations on this topic in the future.

\section{Static wetting on soft substrates}

\subsection{Substrate deformation induced by capillary forces}

\subsubsection{Theoretical analysis}

Numerous theoretical models, which are mostly based on the theory of elasticity, have been developed to describe the substrate deformation near the contact line. The main difference among these models is the way to describe surface traction distribution produced by capillary forces. Lester was probably the first to investigate a liquid droplet at rest on a soft, semi-infinite substrate [10]. In his work, the vertical component of liquid surface tension was modelled as a uniform stress directed upwards and distributed over a narrow region of width $w$ around the TPCL (see Fig. 2a), while the Laplace pressure was distributed uniformly over the liquid-solid contact area of radius $R$. The width $w$ was associated with the capillary layer thickness and assumed to be of an order of nanometer. As the gravitational force is negligible for so small droplet, the integral of this stress distribution over the whole area is equal to zero, i.e., the resultant force in the direction normal to the substrate is zero. This approach of stress analysis was further adopted by Rusanov, who also pointed out that a tangential stress due to the deviation of the static contact angle from the Young's angle should be considered $\left[23^{*}, 24\right]$. The predicted deformation profiles for sessile droplets on semi-infinite and thin elastic substrates were found to agree well with the experimental observation (Fig. 4a \& b) [29]. In the above two models, the vertical substrate displacement near the contact line scales as $L_{e c} \ln (R / w)$, which suggests the divergence of strain as $w \rightarrow 0$, where $R$ is the contact radius. Shanahan and de Gennes stated that the strain singularity can be regularized by postulating a cut-off length $\varepsilon$ (see Fig. 2b), below which the solid material no longer behaves linearly elastic [11]. By modelling the vertical liquid surface tension as a concentrated point force acting on the substrate, Shanahan derived the displacement profile in the region far from the contact line [30,31]. The cut-off length, which strongly affects the substrate deformation profile, is difficult to be determined both theoretically and experimentally. It is typically regarded as an adjustable parameter ranging from angstroms to micrometres in the application of this model [30-32"*].

Deryagin and co-workers proposed modelling the force distribution on the soft substrate using the disjoining/conjoining pressure concept [25]. In [24] the disjoining pressure isotherm was applied to determine the stress distribution in the region between the bulk liquid and the
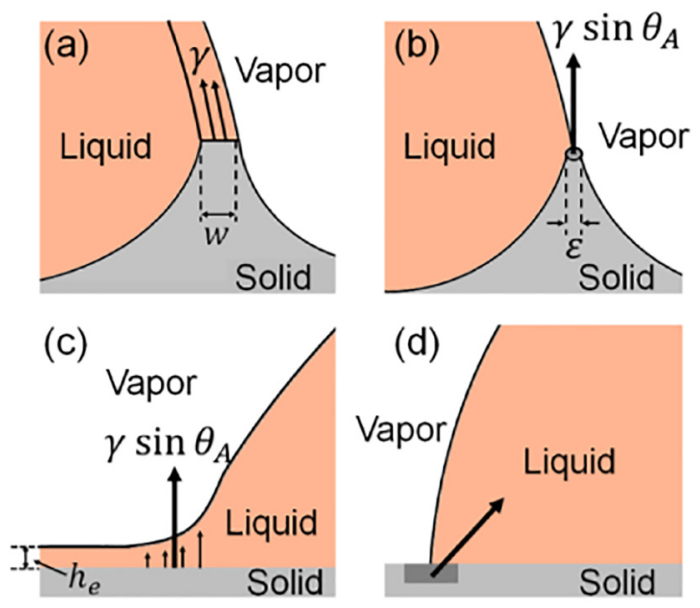

Fig. 2. Scheme of the vertical component of liquid surface tension applied on the soft substrate. (a) The liquid surface tension uniformly pulls on soft substrate over a liquidvapor interfacial region of width $w$. (b) The vertical component of the surface tension, $\gamma \sin \theta_{A}$, is a point force applied on the substrate and a cutoff length $\varepsilon$ is defined in the region of the contact line. $\theta_{A}$ is the apparent contact angle. (c) The vertical component of the surface tension is applied through the disjoining pressure distributed within a transition layer between the bulk liquid and a precursor film of thickness $h_{\mathrm{e}}$. (d) A net pulling force directed inward to the drop is applied on the substrate near the contact line. 
adsorbed precursor film [9], i.e., the region near the apparent threephase contact line as shown in Fig. 2c. The thickness of the precursor film at equilibrium, $\mathrm{h}_{\mathrm{e}}$, was obtained by balancing the disjoining pressure with Laplace pressure, $\Pi(\mathrm{h})=-\Delta \mathrm{P}$. The authors have shown that the integral of the disjoining pressure distribution around the contact line is equal to $\gamma \sin \theta_{e q}$. However, no analytic solution for substrate deformation has been derived in this work. White furthered the work by Deryagin et al. and considered two types of disjoining pressure isotherms: (i) the first leading to the formation of the precursor film in the case of complete wetting and (ii) the second considering a partial wetting scenario without precursor film [33]. An analytical expression for the substrate deformation was derived using the surface Green function. The asymptotic behaviour of deformation has been shown to be independent of the form of disjoining pressure isotherm.

Thermodynamically, the droplet profile and the profile of the deformed soft substrate (see Fig. 3) should satisfy the requirement of minimum excess free energy, independently of the rigidity of the substrate. The excess free energy should include the excess energy terms related to extra (i) liquid-air interface; (ii) soft solid-liquid interface (caused by the deformation); (iii) volume; (iv) surface forces acting near the apparent TPCL [2]; and ( $v$ ) deformation of the soft solid under the action of both capillary and surface forces [34"]. In the equilibrium state, the first variation of excess free energy should be zero, which results in two Euler equations for the profiles of the droplet and the substrate. Solving these two equations yields profiles of the droplet over the soft substrate and of the deformed substrate around and below the droplet. The two conditions for minimizing the excess free energy are well known [35]:

(i) the positive second variation of the excess free energy, which is always satisfied [36];

(ii) the solution of the corresponding two Jacobi equations should satisfy the Jacobi condition [35].

If condition (ii) is satisfied the solution provides the minimum for the excess free energy. On the other hand, if condition (ii) is not satisfied the solution of equilibrium droplet and substrate profiles does not correspond to the excess free energy minimum. To the best of our knowledge no solutions published in literature have been checked for satisfying the Jacobi condition.

Ahmed et al. [34] theoretically investigated sessile droplets in equilibrium on soft substrates. The disjoining pressure near the TPCL was considered and it was shown that the combination of disjoining and capillary pressures determine the substrate deformation. A simplified linear disjoining pressure isotherm and a simple Winkler's model accounting for substrate deformation allowed deriving analytical solutions for the droplet profile and the substrate deformation. The equilibrium contact angle of the liquid droplet with the deformable substrate was also calculated and its dependency on the system parameters was investigated [34"].

Besides the capillary forces from the liquid, the surface tension or interfacial energy of the solid should also have contributions to the substrate deformation. Long et al. derived the theoretical solution for substrate deformation of a thin film by minimizing the total free energy including the surface energy due to the increase of the film surface area and the elastic energy due to its deformation [37]. By considering these energy penalties, they found that the strain singularity close to the contact line can be naturally ruled out. The predicted deformation in the long and short wave vector matches well with experimental observations [29], yet large discrepancies were found at distances from the TPCL comparable to the film thickness. Das et al. analysed the liquidsolid interactions near the TPCL at microscopic level with the use of density functional theory (DFT). The authors suggested that, in addition to the vertical component of surface tension, a tangential force of magnitude $\gamma(1+\cos \theta)$ is applied on the substrate and yields a resultant force directed inwards into the liquid (Fig. 2d) [38]. This capillary force originates from the attractive interactions between liquid and solid molecules, which was then confirmed experimentally [39]. They further calculated substrate displacement profile of small deformations while large deformations will cause a significant effect on the capillary tractions [38]. Jerison et al. proposed that the newly created substrate surface area via its deformation results in an out-of-plane restoring force applied to the substrate $\left[40^{\circ}\right]$. Their theoretical predictions well matched the out-of-plane displacement, while the discrepancies for the in-plane displacement could not be resolved. A similar theory was reported later [41], where a three-dimensional solution for the deformation was derived.

In the above works, the surface stress $Y$ - which refers to the excess mechanical tension inside the interfacial region - replaces the concept of the surface free energy $\gamma_{f}$. For liquids, the new surface created by deformation or stretching is made up by molecules transferred from the bulk liquid to the surface, i.e., the configuration of liquid molecules at the surface does not change and thus the above assumption is valid. The molecules constituting solids and soft solids are not mobile as in liquids and they preserve their relative positions during deformations. The separation between surface molecules, though, changes during deformations and the surface free energy is strain-dependent. Thus, the surface stress is not only dependent on the surface free energy but also on its derivatives with strain, i.e. $Y=\gamma_{f}+d \gamma_{f} / d \epsilon$. This is known as the Shuttleworth effect [42], where $\epsilon$ is the elastic strain. It has been demonstrated that $d \gamma_{f} / d \epsilon$ can be comparable to the liquid surface tension [43"] and its contributions due to substrate deformation should not be neglected. Recent studies showed that the Shuttleworth effect can cause very large strains near the TPCL for drops deposited on a soft film $[44,45]$ and - further - a discontinuity of strain across the contact line was measured for a thin silicone rod immersed in water [46,47]. However, a comprehensive theoretical treatment of capillary-induced substrate deformations including the Shuttleworth effect and how it affects wettability is still missing.

\subsubsection{Numerical simulations}

Since a rigorous theoretical model describing substrate deformation induced by capillary forces for all possible substrate geometries and constitutive laws is still not available, numerical simulations provide an effective way to explore this problem. Madasu and Cairncross simulated the elastic deformation with the nonlinear Mooney-Rivlin constitutive law for incompressible rubber-like solids using a finite element method (FEM) tool [48]. To relieve the strain singularity near the TPCL, two boundary conditions - distribute the line force over a

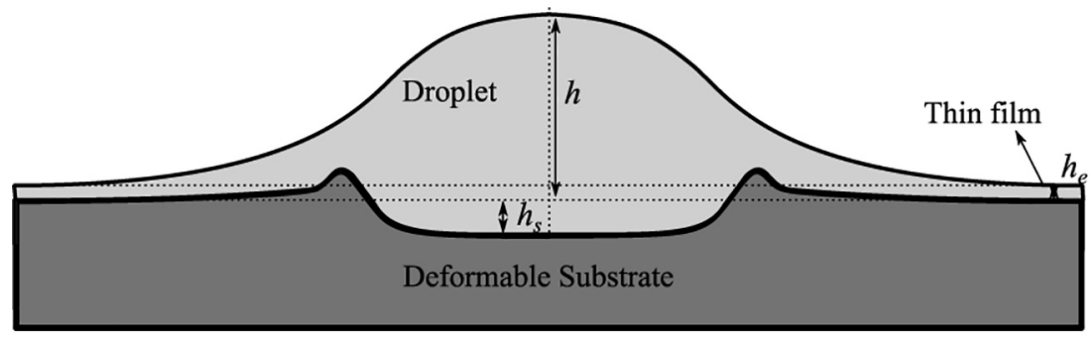

Fig. 3. Schematic diagram of the liquid droplet on a soft substrate. 
macroscopic region and fix the crease angle of the solid - were used. The numerical solutions suggest that surface displacement is dependent on liquid surface tension and substrate shear modulus, like predicted by the analytical models. Using the same approach, Yu et al. investigated the effects of substrate thickness on the elastic deformation caused by a millimetre-sized droplet [49]. They found that the maximum vertical displacement decreased with increasing thickness when the substrate was thinner than $\sim 2 \mathrm{~mm}$, while the elastic deformation on thicker substrates behaved like that on semi-infinite substrates. They further compared the simulation results obtained with the nonlinear Mooney-Rivlin constitutive law and a linear elastic constitutive model, and concluded that the simple linear elastic model is accurate enough to describe elastocapillary deformation [50]. Indeed, a number of numerical works have reported that the simulated deformation profile using the linear elastic model matches well with the experimental observation (e.g., Fig. 4a \& b) [20,29,51*0. However, the numerical models used in $[49,50]$ are based on the assumption that the contact angle is not affected by substrate deformation. In addition, the upward force acting in the contact line region (the vertical component of liquid surface tension) is modelled as a uniform force acting on the contour of the circular contact region. It is not evident what the thickness of the ring-shape area is over which this force is distributed. Most recently, Gielok et al. investigated the elastic deformation of a soft substrate by a sessile droplet using FEM calculations coupled with lubrication approximation [52]. The disjoining pressure approach was applied to model the surface traction on the substrate and the effects of material stiffness, substrate size and the parameters of the disjoining pressure model on deformation profile were explored. The three parameters of the disjoining pressure isotherm used in [52] determine the equilibrium contact angle, the adsorbed film thickness and the length scale $a$. This scale, which is of the order of the capillary layer thickness $w$ mentioned in the work of Lester [10], is the width of the transition region between the macroscopic droplet and the adsorbed film where the influence of the disjoining pressure is significant. In [52] the parameters of disjoining pressure have been chosen in such a way that the equilibrium contact angle did not change. It was shown that the dimensionless maximum displacement scales with the dimensionless $L_{e c} \sin \theta / a$ and the solid angle of the deformed substrate near the contact line scales with $L_{e c} / a$. In agreement with [49] it was confirmed that the substrate deformation decreased with substrate thickness, whereas the influence of the substrate thickness on the deformation near the TPCL was weaker than near the droplet centre.

Based on continuum mechanics, numerical approaches such as FEM are capable to capture the main features of wetting ridges on deformable substrates. However, the microscopic liquid-solid interactions, which take place at the molecular scale, are still elusive. Molecular dynamics (MD) simulations were therefore applied to investigate the microscopic mechanisms. Léonforte and Müller investigated the wetting of polymer nanodroplets on deformable substrates [53]. They found that the formation of the wetting ridge is not only dependent on the stiffness of the substrate, but also on the compatibility between the liquid and the solid (i.e., the wettability). Moreover, the height of the wetting ridge is reduced by the line tension $\kappa$ for sufficiently small droplets. Their results suggest that at the sub-micro- or nanoscale additional forces due to size-dependent effects, such as the line tension, must be considered in modelling capillary phenomena. Similar effects were first observed for small droplets wetting non-deformable substrates, for which the line tension was found to affect the equilibrium contact angle $[54,55]$. Weijs and co-workers comparatively studied substrate deformation by a nanodroplet and a nanobubble using MD simulations [47]. They showed that the local displacement of the solid near the contact line always points inwards the liquid, i.e. the more dense phase, which confirms the theoretical analysis by Das et al. [38]. These results indirectly also demonstrated that the surface stress is different from the surface free energy, i.e. the Shuttleworth effect.

\subsubsection{Experimental observations}

In the literature, capillary-induced substrate deformations were studied via various experimental approaches. In early works, imaging techniques such as scanning electron microscopy [56], transmission electron microscopy [57], atomic force microscopy [13,58] and optical microscopy [13] were employed. However, these techniques could not provide quantitative data of the deformation profiles. Using the scanning white-light interferometric microscopy, Carré et al. obtained the profile of the wetting ridge generated by droplets resting on deformable substrates $\left[14^{\circ}\right]$. They showed that the surface displacement in the air phase matches well with the theoretical prediction one by Shanahan's model [11,26,30,31]. However, they did not manage to image the deformation of the solid-liquid interface. Applying an optical profilometry, $\mathrm{Pu}$ et al. analysed the entire profile of the wetting ridge near the TPCL
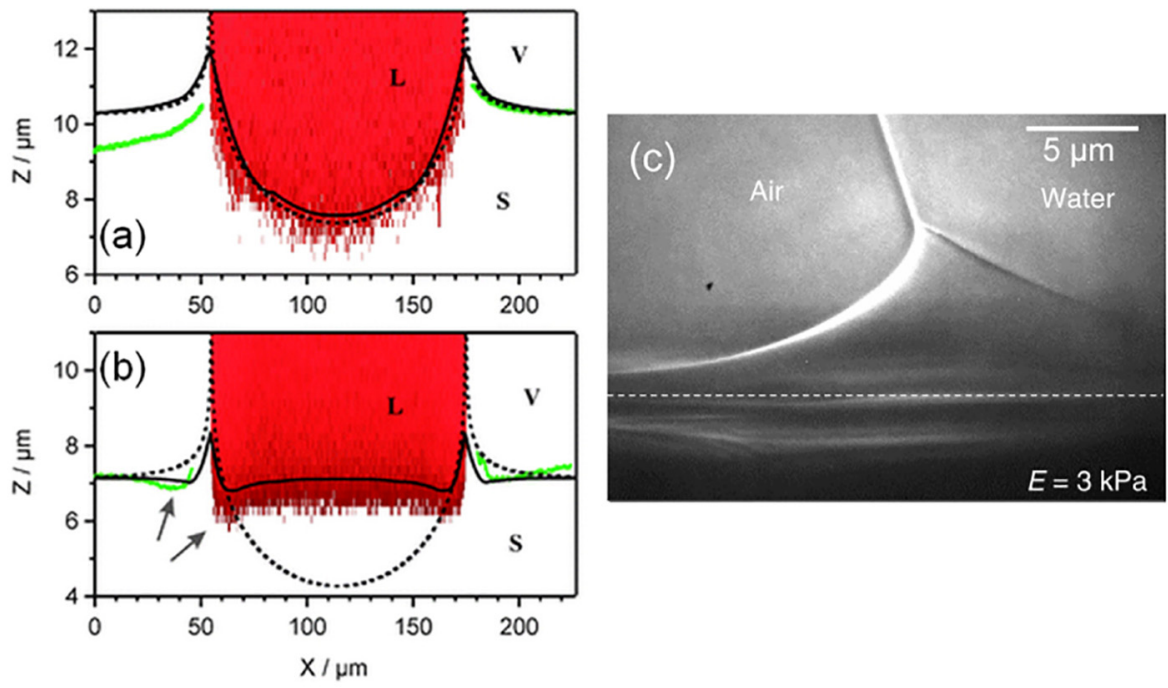

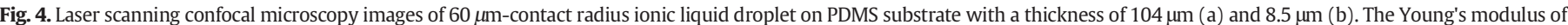

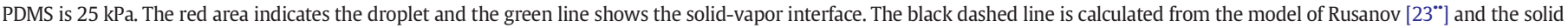
black line is a profile simulated by FEM. Reproduced with permission from Ref. [29]. (c) X-ray image of a wetting ridge formed on a silicone gel with a Young's modulus of 3 kPa. Reproduced with permission from Ref. [65]. S, V and L in (a)-(b) indicate the solid, vapor, and liquid regions, respectively. 
formed in a series of experiments, including dip-coating [59-61], sliding [62] and evaporation [63] of sessile droplets on soft viscoelastic substrates. It was found that substrate deformation manifests its shape and profile as changes in the contact angle as the droplet volume is increased or reduced. Moreover, a second order structure with a height of few hundreds nanometers were observed at the centre of ridge peak [60], which they believed was the cut-off region discussed by Shanahan and de Gennes [11]. However, in these experiments the droplets were removed from the substrate prior to imaging, and thus the measured profiles do not reflect the original substrate deformation since the substrate started recovering its flat profile soon after removing the droplet.

Pericet-Camara et al. characterized the entire deformation profile of soft polydimethylsiloxane (PDMS) substrates below a microdrop of ionic liquid by means of laser scanning confocal microscopy (LSCM) [51“]. The droplet was dyed with the fluorophore Nile red, which can be excited by a He-Ne laser with a wavelength of $543 \mathrm{~nm}$. Threedimensional images of the fluorescing droplet and the deformed substrate were obtained by recording the fluorescent and the reflected light signals simultaneously but separately, and later combining them in one image. Their results showed for the first time directly that in addition to the wetting ridge near the contact line a dimple is formed underneath the droplet due to the Laplace pressure (Fig. 4a). They further investigated the effects of substrate thickness on elastic deformation [29]. It was shown that both the wetting ridge at the droplet rim and the dimple underneath the droplet are affected by the solid support below the elastic film if the film is thinner than a critical value (Fig. 4b), which is $\sim 50 \mu \mathrm{m}$ for soft PDMS with $G \sim 25 \mathrm{kPa}$. Jerison and co-workers embedded two layers of fluorescent beads in an elastic substrate, one at the free surface and the other below the surface, and the entire deformation profile of the substrate was imaged by tracing the fluorescent particles using confocal microscopy [40"]. Employing the same technique, Style et al. showed that the substrate deformation follows a universal law close to the TPCL that is entirely determined by the liquid properties and that the profile is independent of droplet size and substrate thickness [64"]. The authors investigated a droplet-surface system with symmetric surface tensions, i.e., the liquid-solid surface tension equals to the liquid surface tension and a symmetric profile was observed. Most recently, Park et al. directly visualized the tip region of the wetting ridge of a water droplet on a silicone gel by X-ray microscopy [65]. Compared to the other techniques mentioned above, this technique can precisely measure the geometry and formation process of the cusp tip where liquid, solid and vapor meet. Due to the asymmetric surface tensions of the system, they observed an asymmetric cusp shape (Fig. 4c). However, they found that the displacement profile at a distance from the TPCL (much larger than the elasto-capillary length) can still be well described by the symmetric model developed by Jerison et al. $\left[40^{\circ}\right]$.

\subsection{Effects of substrate deformation on contact angle and contact angle hysteresis}

Wetting on rigid substrates is solely governed by the surface free energies of the liquid-solid-vapor interfaces [1,9], and the contact angle can be described by the Young's equation or the modified Young's equations (i.e. Cassie and Wenzel equations). In contrast, on soft substrates the elastic deformation is an additional term affecting surface wettability. From the perspective of mechanics, the wetting ridge lifts the TPCL and thus a new balance of three interfacial tensions is established. Thermodynamically, the increase of substrate surface area because of substrate deformation leads to a free energy penalty of the system. As a result, the apparent contact angle measured on a flat soft substrate may differ from the Young's angle (Fig. 1).

The contact angle of liquid droplets on deformable substrates has been investigated theoretically and experimentally. Based on the theoretical solution of the deformation profile, Lester obtained a simple relationship between the apparent contact angle and the angle between the deformed substrate and the horizontal plane using Neumann's law $\left[10,12^{\circ}\right]$. He concluded that Young's equation is still useful to describe the apparent contact angle on sufficiently rigid substrates, but a larger apparent contact angle will be obtained on softer substrates. Indeed, this was confirmed by early experimental work of Soon and Mu [66] and more recently by a number of experimental studies [20,21]. Adopting the same analytic method, also Rusanov $\left[23^{*}, 24\right]$ and Shanahan $[30,31]$ derived the mathematical expression of the apparent contact angle on soft substrates. However, their models predict that the apparent contact angle is smaller than the Young's angle. Marchand et al. stated that the contact angle on deformable substrates can be described neither by Young's equation for perfectly rigid substrates nor by Neumann's law for purely viscous liquid substrates [67]. They demonstrated that the transition of the contact angle from the rigid limit to the soft limit takes place when the elastocapillary length reaches the molecular scale. Style et al. argued that the effect of substrate stiffness on the apparent contact angle is also dependent on droplet size $R$ [41]. In their work, three wetting regimes have been identified theoretically:

(i) if $R \gg Y_{S} / G$, the substrate behaves like a rigid one and Young's equation holds;

(ii) if $R \ll Y_{S} / G$, the substrate responds as a liquid and Neumann's law describes the wetting system;

(iii) if $R \sim Y_{S} / G$, the formed wetting ridge serves as an effective line tension making the substrate less wettable and leading to an increased contact angle.

However, many experimental studies have reported that the contact angle of millimetre-sized droplets on soft substrates with similar surface stress increases with decreasing substrate stiffness $[20,21,32 * 68]$, indicating that Young's equation is not applicable for droplets with radius much larger than the elastocapillary length, i.e., $R \gg L_{e c}$. Moreover, Style et al. have observed a decrease of the contact angle with decreasing $R$ (i.e., for the case of $R \rightarrow Y_{S} / G$ ) in an experimental work [64"], which further disagrees with their theoretical model. Lubbers et al. suggested that the equilibrium shape of a liquid droplet on an elastic substrate is actually governed by three length scales, namely the size of the droplet $(R)$, the elastocapillary length $\left(L_{e c}\right)$, and the characteristic length for molecular interactions $(a)\left[69^{\circ}\right]$. They found that the apparent contact angle only changes when $L_{e c} / R \gg 1$ and the microscopic contact angle differs from Young's angle when $L_{e c} / a \gg 1$. A complex dependence of the apparent contact angle on $L_{e c} / a$ was also reported by Gielok et al. in their numerical simulation work [52].

Another key descriptor of surface wettability is the contact angle hysteresis $(\mathrm{CAH})$, defined as the difference between advancing and receding contact angle [1]. For rigid substrates, surface roughness and chemical heterogeneity are the cause of contact angle hysteresis [8, 70]. For soft substrates, the wetting ridge formed near the TPCL is considered as the main source of pinning of the contact line and leads to a large $\mathrm{CAH}$. Since the height of the wetting ridge is inversely proportional to the substrate stiffness, the CAH on rigid substrates should be smaller than that on soft substrates. This has been confirmed by numerous experiments in literature $[13,20,21,71]$. Recent experiments also revealed that the wetting ridge can adjust its profile as the apparent contact angle changes [61-63]. Consequently, the dynamic contact angle hysteresis is a function of the speed of the moving contact line [72]. Lee et al. have shown that the advancing and receding contact angles of a water droplet on a soft bitumen substrate determined by slowly increasing/decreasing the volume of a sessile droplet strongly depend on the rate of the volume change of the droplet (volume flow of blowing/sucking) [73].

The combined action of capillary pressure and surface forces acting near the apparent TPCL and the elasticity of the substrate determine the shape of the liquid meniscus and the substrate deformation [74]. 
Most recently, a theory of CAH of sessile liquid droplets on soft/deformable substrate was developed in terms of disjoining pressure isotherm, $\Pi(h)[2]$, accounting for the action of surface forces near the TPCL [74]. The excess free energy calculation for a droplet deposited on a soft substrate yields two equations for droplet and deformed substrate profiles in terms of the disjoining pressure isotherm. A simplified sshaped disjoining pressure isotherm, allowing direct calculations of static, advancing and receding contact angles on soft substrates, was adopted. Elasticity of the substrate was assumed to obey a simple Winkler's model for elastic surfaces [74]. The obtained results are in an agreement with the CAH theory developed for non-deformable substrates [ $\left.75^{\circ}\right]$. Calculated advancing and receding contact angles were found depending on droplet volume and were validated experimentally. Furthermore, they were lower on deformable substrates than that on non-deformable substrates $\left[75^{\circ}\right]$.

\section{Dynamic wetting on soft substrates}

\subsection{Spontaneous wetting}

When a liquid droplet contacts a solid substrate, the unbalanced capillary force, $\gamma\left(\cos \theta-\cos \theta_{e q}\right)$, drives the liquid to spread on the substrate until it reaches equilibrium. On rigid substrates, three distinct spreading regimes, which reveal different types of resisting forces, have been identified for low viscosity droplets. Just after contact between the droplet and substrate, the spreading is resisted by inertial force and the spreading radius, $r$, grows with time, $t$, according to a power law $r \sim t^{0.5}$, regardless of liquid and surface properties [76]. This wetting regime normally lasts less than $\sim 0.1 \mathrm{~ms}$ and then the spreading enters another, slower regime, in which the dynamics is still dominated by inertia but depends on surface wettability. The wetting follows another power law, $r \sim t^{\alpha}$, where $\alpha$ increases from $\sim 0.25$ for $\theta_{e q} \approx 120^{\circ}$ to $\sim 0.5$ for $\theta_{e q} \approx 0$ [77-79]. As spreading proceeds further, the spreading speed decreases and the inertial force lessens. At $t \gtrsim 10 \mathrm{~ms}$ (depending on liquid properties), the viscous friction force within the droplet becomes the main factor resisting spreading, and the wetting follows the Tanner's law, $r \sim t^{0.1}[1,9]$. In general, wetting of low viscosity liquids on rigid substrates follows different power laws at different timescales.

As discussed in the previous sections, a sessile droplet sitting on a soft substrate forms a wetting ridge around its contact line. If the droplet spreads, the wetting ridge may move with the contact line and thus cause additional energy dissipation, which affects spontaneous wetting. Stapelbroek et al. comparatively investigated the dynamic wetting of water droplets on soft substrates, micro-structured substrates and chemically patterned substrates [80]. They found that the spreading at $t \lesssim 0.1 \mathrm{~ms}$ on all types of substrates follows the same power law of $r \sim t^{0.5}$ as on rigid ones. Chen et al. studied the spreading of various liquids on soft viscoelastic substrates with $G$ between 0.2 and $510 \mathrm{kPa}$ (measured at $1 \mathrm{~Hz}$ ) [68], and have identified two wetting regimes. In the first few milliseconds, the spreading is fast (the speed is $\sim 0.5 \mathrm{~m} / \mathrm{s}$ ) and follows the same power law as on rigid substrates, i.e. the spreading is still dominated by inertia. Then, wetting enters a slower regime with a spreading speed of the order of $\sim 10^{-3} \mathrm{~m} / \mathrm{s}$. The slow spreading in this regime was ascribed to the elastic deformation of the soft substrates. As the contact line moves forward, the formed wetting ridge moves with it and causes additional viscoelastic energy dissipation. By balancing the capillary force with the viscoelastic friction force, the spreading law of this regime was obtained, $r \sim\left(1-e^{-\beta t}\right)$, where $\beta$ is a coefficient [32*]. At $t \gtrsim 1 \mathrm{~s}$, Shanahan, Carré and co-workers observed an even slower wetting of low viscosity droplets on viscoelastic substrates with a speed of $\sim 10^{-4} \mathrm{~m} / \mathrm{s}\left[14^{*}, 15,17,30,31,81\right]$. Based on energy balance, they derived another wetting power law, $\left(\cos \theta_{e q}-\cos \theta\right)$ $\sim\left(\frac{U}{U_{0}}\right)^{\alpha \prime}$, where $U$ is the spreading speed and $U_{0}$ is a characteristic speed related to the rate dependent viscoelastic dissipation of the soft material. The exponent $\alpha^{\prime}$ was found to be of the order of 0.5 0.6 in experiments $\left[14^{\circ}, 15,17,81,82\right]$. In summary, the early dynamic wetting of soft substrates by low viscosity liquids is still predominated by inertial force while viscoelastic friction force is main source resisting spreading in later wetting regimes.

Several studies were also conducted to investigate wetting of hydrogel substrates, which are strongly deformable and swollen when they are in contact with liquids. Due to the hydrophilicity of such a substrate, a droplet of water or of an aqueous solution spreads quickly into a thin film and the dynamics is dominated by viscous friction force within the liquid, which follows the Tanner's law [83,84]. For pure water, the droplet is then pinned by the local substrate deformation near the contact line [85]. With further diffusion of water into the substrate, the contact angle of the droplet decreases while the angle of the substrate deformation near the contact line increases. Once these two angles are almost equal, the contact line depins and starts to recede [86]. By comparison, droplets of aqueous surfactant solutions begin to retract immediately after spreading. Moreover, a step-like front with a constant shape was observed to propagate on the substrate in front of the contact line if the substrate is sufficiently soft [85].

The ridging effects on droplet spreading were also reported in dynamic wetting of liquid metals on rigid substrates in high temperature systems. Compared to the wetting of Newtonian liquids on soft substrates, this type of wetting is much more complicated not only because liquid metals are typically non-Newtonian fluids, but also because the process is always accompanied with chemical reactions (e.g. alloy formation), heat and mass transfer. The wetting dynamics has been roughly divided into four regimes [58]. In early times, the elastic deformation due to capillary force is negligible ( $\delta \ll 1 \mathrm{~nm}$ ) and droplet motion is mainly controlled by the viscosity of the liquid metals as that of Newtonian fluids. When the substrate heats up to the softening temperature, a small ridge is formed near the contact line. The spreading proceeds with the movement of the ridge and the contact angle approaches to the Young's angle. In third regime, the ridge grows rapidly with a speed compared with the spreading speed and its size becomes comparable with the droplet size. Finally, at longer times more complex geometries will be developed and the system equilibrates with large deformation of the substrate. Details about wetting of liquid metals could be found in several review articles [87-89].

\subsection{Forced wetting and dewetting}

In practical applications, such as spray coating and crop spraying, liquid droplets are deposited on solid substrates with a certain speed. Therefore, it is important for surface engineers to understand the influence of substrate properties on the impact dynamics and develop strategies to control droplet deposition. Chen et al. performed a systematic investigation of droplet impact on soft viscoelastic substrates and compiled a phase diagram illustrating the dependence of impact outcome on the Weber number ( $W e=\rho R_{0} V_{0}^{2} / \gamma$, where $V_{0}$ is the impact velocity) and substrate shear modulus [71]. As shown in Fig. 5a, droplets can completely rebound from soft substrates when the Weber number is between a lower and an upper threshold $(0.15 \lesssim W e \lesssim 3.5)$, beyond which the droplet is deposited. Droplet rebound was ascribed to the formation of an air film at the solid-liquid interface during impact [16], which has been visualized experimentally [90]. However, the formation and collapse mechanism of this air film is still not well understood. At intermediate Weber numbers $(3.5 \lesssim W e \lesssim 9)$, the generation of a capillary wave upon impact leads to the formation of an air cavity at the drop centre [91], and thus two types of bubble entrapment depending on substrate stiffness have been identified. On soft substrates, the air film underneath the droplet prevents the air cavity contacting the substrate and eventually a bubble is entrapped inside the impinging droplet (see top image in Fig. 5a). On rigid substrates, on the other hand, the air cavity touches the substrates during drop retraction and consequently a bubble is entrapped on the substrate after impact (see top image in Fig. 5a). At high Weber numbers (We $\gtrsim 9$ ), partial rebound, i.e. the 

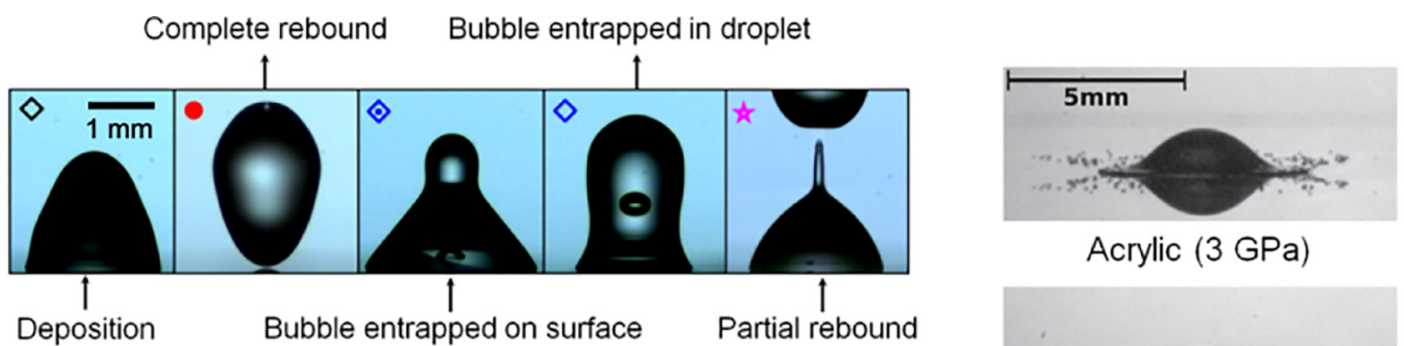

Acrylic (3 GPa)
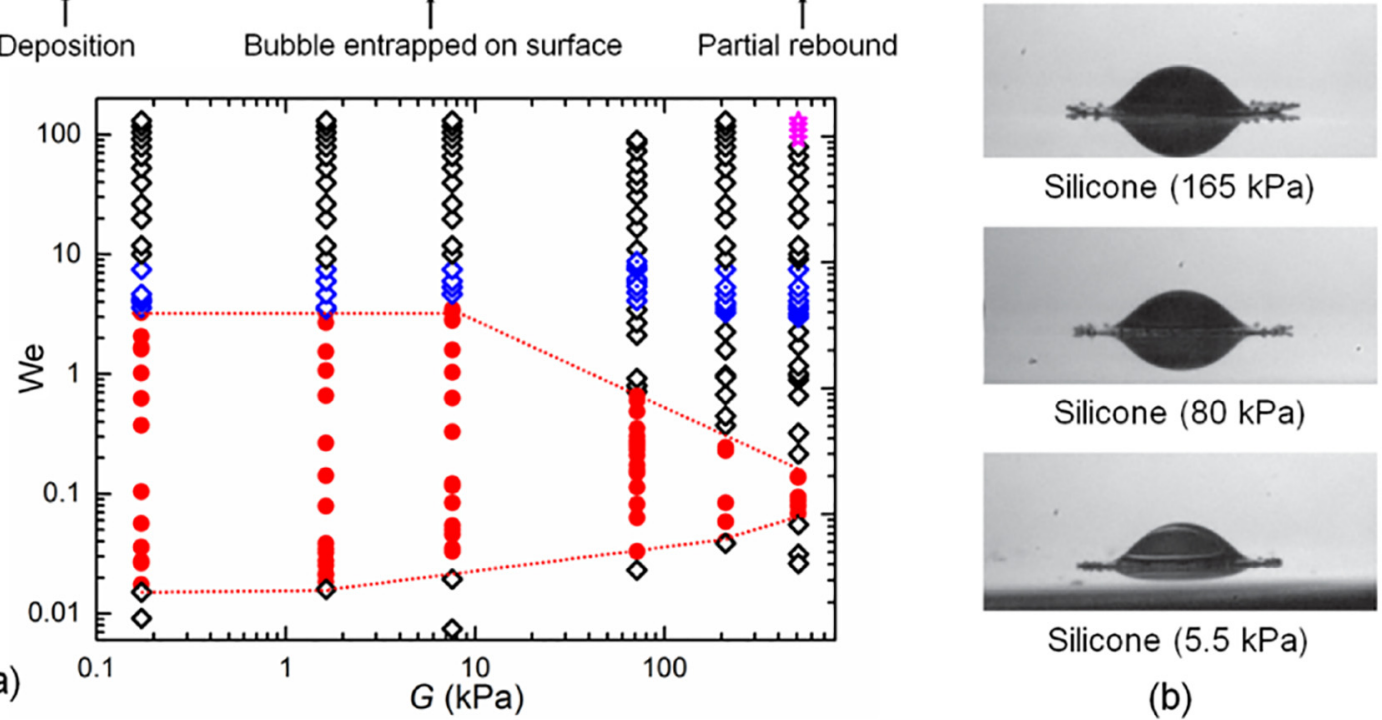

Silicone $(165 \mathrm{kPa})$

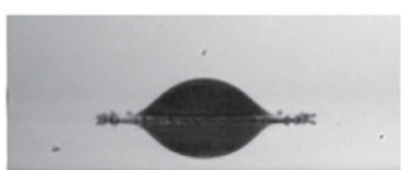

Silicone $(80 \mathrm{kPa})$

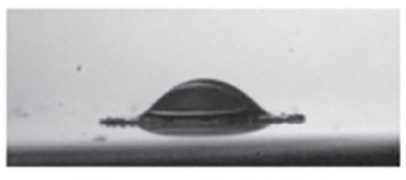

Silicone $(5.5 \mathrm{kPa})$

(b)

Fig. 5. (a) Impact phase diagram of water droplets impinging on soft viscoelastic substrates as a function of Weber number We and shear modulus $G$.

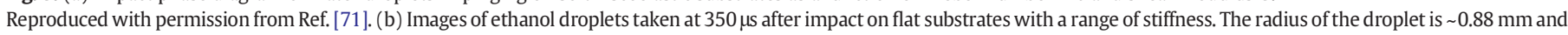
the impact speed is $\sim 2.61 \mathrm{~m} / \mathrm{s}$. Reproduced with permission from Ref. [92*].

breakup of the impinging droplet into two parts, was observed on rigid substrates [71]. Recently, Howland et al. reported that substrate stiffness can also affect the threshold velocity for splashing at Weber numbers up to 260 and Young's moduli as small as $5 \mathrm{kPa}$ (Fig. 5b) [92]. The substrate deformation, which is developed during impact and can be up to few tens of micrometres [93], dissipates parts of the kinetic energy of the moving droplet and thus reduces splashing.

The analysis of the contact line dynamics during impact reveals that substrate stiffness affects the impact process in a nontrivial way. For bouncing droplets (i.e., non-depositing or non-wetting), the spreading and retracting processes are independent on substrate stiffness, as soft substrates show a higher effective stiffness during impact and rebound [71]. The same conclusion has been made for the spreading stage of wetting droplets $[93,94]$. In contrast, the retracting stage of wetting droplets depends more decidedly on substrate stiffness. At low Weber number, an air film is formed between soft substrate and droplet and thus the droplet retracts as a bouncing droplet until the air film breaks down; on the other hand, at the same Weber number no air film is formed on a rigid substrate and so the droplet does not retract from the substrate. At high Weber numbers, the wetting ridge formed on soft substrates pins the droplet contact line at the maximum spreading radius and suppresses drop retraction. By comparison, on rigid substrates at the same Weber numbers the small wetting ridge moves with the receding contact line. Droplet retraction is thus slower on less stiff substrates due to larger energy dissipation $[93,94]$. The pinning of the contact line by the wetting ridge also leads to a linear dependence of the contact angle hysteresis on the impact velocity [72].

Contact line dynamics on soft substrates was also investigated by other approaches of forced wetting and dewetting. Using the Wilhelmy plate method, Pu and Severtson observed a stick-slip behaviour of the contact line on viscoelastic substrates as a result of the pinning effects of the wetting ridge [59-62,95]. The pinning force is of the order of few millinewtons and determined by the liquid surface tension [61, 95], the contact line speed [61] and the thickness of the soft substrate
[59], which all influence the extension of the wetting ridge. The stickslip behaviour of the advancing or receding contact line was also reported in contact angle measurements with sessile drops on viscoelastic gels [96-98] and on viscoelastic bitumen surfaces [73]. It can only be observed when contact line speed is between a lower and an upper limit, beyond which the contact line moves continuously. These limit speeds are set by the dynamic rheology properties of the substrates: substrates response as viscous liquids when the contact line speed is below the lower limit and as purely elastic solids when the contact line speed is higher than the upper limit [98].

\section{Other capillary phenomena on soft substrates}

\subsection{Sessile droplet evaporation}

Lopes and Bonaccurso investigated the evaporation of water droplets on soft, elastic PDMS substrates with different Young's moduli [20]. Like on most rigid substrates, three successive evaporation modes, namely, the constant contact radius (CCR), the constant contact angle (CCA) and the mixed mode, have been identified. They have shown that substrate stiffness affects substrate deformation, and thus the receding contact angle and the transition from CCR to CCA evaporation. On stiffer substrates, this transition occurs earlier and evaporation takes longer to complete; on less stiff substrates, contact line pinning due to the more pronounced wetting ridge traps the droplet for a longer time in the CCR evaporation mode, and thus the drop evaporates faster. The authors further applied this finding to use substrate stiffness for controlling colloid particle deposition via droplet evaporation [21]. On viscoelastic PDMS substrates, Yu et al. observed a short stick-slip regime after CCR evaporation, in which the contact radius decreases while the contact angle increases [99]. Pu and Severtson comparatively studied droplet evaporation on viscoelastic PDMS substrates and acrylic thermoplastic substrates [63]. In contrast to multi-evaporation modes observed on PDMS substrates, water droplets evaporated with 
continuous expansion of the contact area on acrylic polymer substrates. This new evaporation mode was attributed to the relaxation of the substrate deformation and the outward action of the capillary pressure [63].

By depositing solvents on soluble polymer substrates, Bonaccurso et al. fabricated microstructures via droplet evaporation [100,101]. The formation process of the microstructures can be briefly described as follows. After the soluble droplet is deposited on the polymer substrates, it spreads out and forms a flat lens. The solvent diffuses into the polymer, softens it and causes the formation of a gel-like surface layer. This soft layer is further deformed by the surface tension pulling upwards at the contact line and the Laplace pressure pushing downwards at the liquid-solid contact area. Eventually, a spherical crater with a rim near the ridge is formed after the solvent is completely evaporated.

\subsection{Dropwise condensation and frost growth}

Sokuler et al. performed an experimental study of dropwise condensation on viscoelastic substrates with different stiffness [22]. They found that nucleation is favoured on softer substrates, i.e., the nucleation rate is faster, and observed a higher nucleation density as well (Fig. 6a). This behaviour was attributed to a lower nucleation barrier on soft substrates due to a lower free energy of condensation that is coming as a result of substrate deformation around the small liquid condensed nucleus. The authors further showed that the viscoelastic nature of the material leads to a longer relaxation time for merging droplets on a soft substrate than on a rigid substrate, and the merging can even be inhibited on very soft substrates [22]. Following these experiments, Eslami and Elliott compared heterogeneous nucleation on a viscous and a rigid substrate of similar surface free energy [102]. Their thermodynamic analysis demonstrated that the energy barrier for heterogeneous nucleation on a viscous substrate is indeed lower than on a rigid substrate, which is due to the "deformability" of the viscous liquid. It is noted that the Gibbs free energy of nucleation of droplets on a viscoelastic substrate is more complicated than that on a viscous liquid substrate since an extra elastic energy term of substrate deformation needs to be considered. Thus, a rigorous model for condensation/heterogeneous nucleation on viscoelastic substrates is still missing. Style et al. condensed water droplets on soft elastic films with a thickness gradient supported by a much stiffer substrate [103]. They found that all nucleated droplets moved to the "softer" region on the film, i.e., to that where the film is thickest and thus the influence of the supporting stiff substrate is weakest (Fig. 6b). This phenomenon is like the so-called "durotaxis" behaviour of living cells, who actively sense stiffness differences on substrates they are placed on and move accordingly. Durotaxis for droplets was rationalized by the effect of substrate stiffness/thickness on contact angle. It is known that the contact angle decreases with substrate thickness $\left[41,64^{\circ}\right]$. As a result, on soft substrates with a thickness gradient, droplets have asymmetric contact angles, i.e., the contact angle is large on the thin/rigid area and small on the thick/soft area. This causes a net capillary force directed towards the soft region, which activates the movement of the condensed droplets [103]. Most recently, Phadnis and Rykaczewski experimentally and numerically investigated the effects of substrate stiffness on dropwise condensation heat transfer [ $\left.104^{\circ}\right]$. It was found that soft substrates with a shear modulus below $500 \mathrm{kPa}$ can significantly reduce the condensation heat transfer rate, which is primarily driven by additional thermal resistance of the liquid posed by depression of the soft substrate. This finding suggests that although the nucleation rate is high on soft substrates, they may not be better for condensation compared to rigid substrates from the perspective of droplet life cycle.

Substrate stiffness does not only influence dropwise condensation dynamics, but also the subsequent frost growth at low temperatures, as reported by Petit and Bonaccurso [19]. It was found that condensation frosting proceeds via a two-stage growth of dendritic ice bridges (top figure in Fig. 6c), which is a universal behaviour and independent of substrate stiffness. However, substrate stiffness affects the growth velocity of the frost dendrites and the area density of condensed supercooled droplets. Their combined effects determine that the (a)

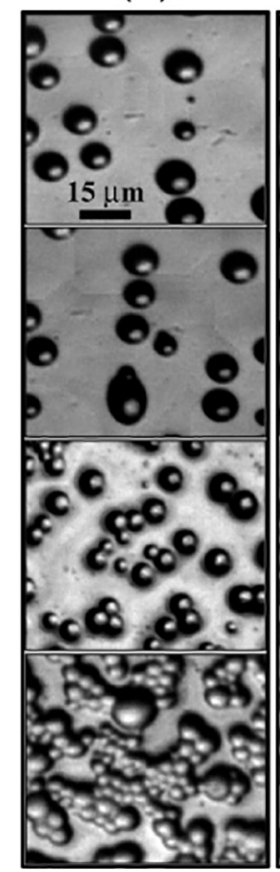

(b)

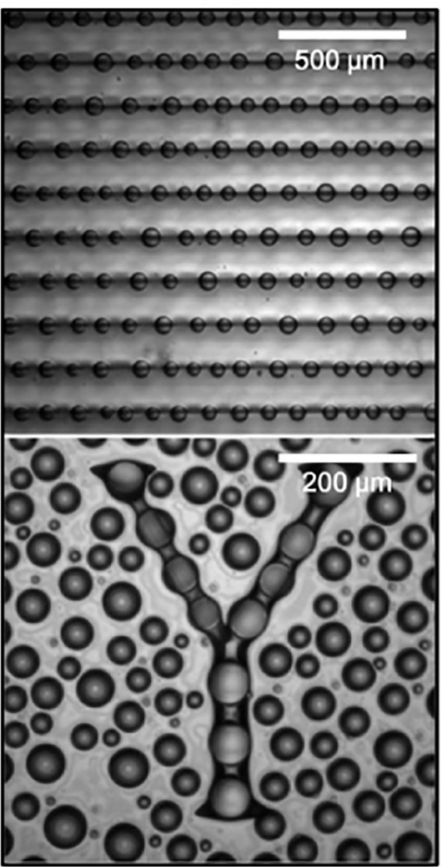

(c)

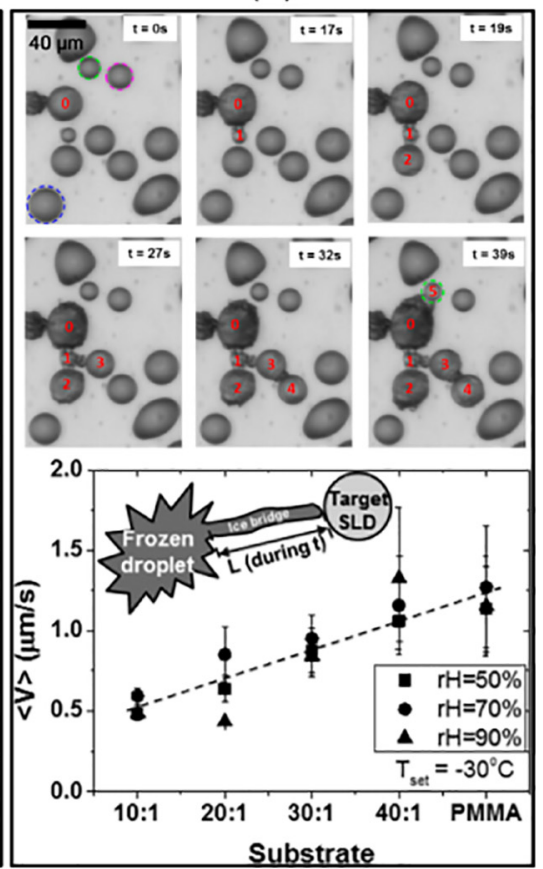

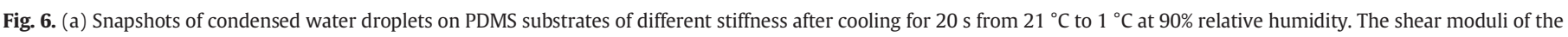
substrates from top to bottom are $50 \mathrm{kPa}, 10 \mathrm{kPa}, 1 \mathrm{kPa}$ and $0.1 \mathrm{kPa}$, respectively.

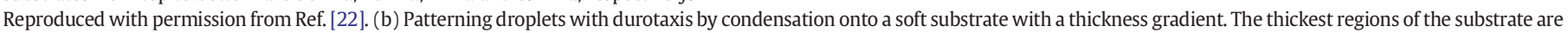

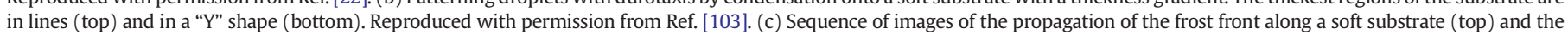
average velocity of ice bridges is plotted as a function of the mass ratio of PDMS monomer and cross-linker (bottom). Reproduced with permission from Ref. [19]. 
freezing time on rigid substrates with low condensation density is longer than that on soft substrates with high condensation density (bottom figure in Fig. 6c) [19], i.e. the frost formation is delayed.

\subsection{Capillary phenomena on "soft" thin films, cantilevers and fibres}

In recent years, several authors have discussed that otherwise "rigid" materials, such as metals and polymers, exhibit "soft" properties if one of their length scales shrinks down to micro- or nanometers [105-108]. Py and co-workers investigated the evaporation of water droplets on a free-standing film with few hundred micrometres thickness $[106,108]$. As shown in Fig. 7a, the vertical component of liquid surface tension bends the corners of the thin film and the droplet can be even completely wrapped up as it evaporates [106]. The complete wrapping can only be observed for films with a length larger than the elasto-capillary length for films $(B / \gamma)^{0.5}$ (where $B$ is the bending stiffness) $[105,106,108]$ and the folding direction is determined by the magnitude of the folding torque [107]. Applying an alternating current electric field, Wang et al. controlled the vertical surface tension component, and thus the wrapping and unwrapping of a sessile droplet by the stimulated vibrating film [109]. Yuan and Zhao further demonstrated that the wrapping and unwrapping processes are also related to the propagation of the precursor film [110] and capillary wave [111]. On the other hand, the Laplace pressure causes the film to bulge outwards by tens of microns, which has been recognized as a new strategy to measure the solid-liquid interfacial tension [112]. For ultrathin films floating on liquid, instead of folding up, a liquid droplet leads to the formation of radial wrinkle pattern near the contact line (Fig. 7b) [113-115]. This winkle pattern is generated due to the radial stress of the surface tension and its geometry is controlled by the elasticity and thickness of the films.

Elastic microbeams or microfibers can also be easily deflected by capillary forces.

For example, a microdrop deposited on a rectangular cantilever of atomic force microscope bends up the cantilever by typically a few hundred nanometers $\left[116{ }^{*}, 118,119\right]$, as shown in Fig. 7c. The elastic response of the cantilevers allows to monitor capillary phenomena in small scales, such as the evaporation of microdrops [116“"119-121], the diffusion of liquid molecules into polymers [122,123] and also the wetting dynamics of microparticles [124]. While a droplet is deposited between two flexible fibres, it can completely spread, partially spread or not spread along the fibres, depending on the competition between elasticity and capillarity (Fig. $7 d$ ) [117"]. The subsequent evaporation of the droplet deposited between two fibres can cause the droplet to spread to form a liquid column under the capillary forces and thus the evaporation rate is enhanced [125].

\subsection{Capillary phenomena on slippery liquid-infused substrates}

Slippery liquid-infused substrates, which are fabricated by infusing a lubricating oil into textured, porous solid substrates, have attracted considerable attention recently $[126,127]$. These types of substrates are inspired by natural plants like the Nepenthes pitcher plant and have excellent liquid-repellent properties. Since the lubricating oil is the functional layer, the substrate is also a special type of "soft" substrate. When a liquid droplet is deposited on such a substrate, a wetting ridge is as well formed under the action of surface tensions (air-oil, air-liquid, and liquid-oil), and the droplet can even be completely cloaked by the oil if the balance of surface tensions is favorable [128*"]. The cloaking film is several tens of nanometers thick and acts as a barrier against the diffusion of vapor molecules during dropwise condensation, lowering the performance of condensation heat transfer $[129,130]$. If the balance of surface tensions is not favorable, droplets are not cloaked by the oil. In this case, condensate droplets as small as $100 \mu \mathrm{m}$ are highly mobile and the remarkable mobility leads to a sweeping effect that clears the surface from droplets and allows for fresh nucleation thus enhancing condensation [129]. It was also found that the viscous dissipation in the wetting ridge is the main source dissipating the gravitational potential energy of sliding droplets on an inclined substrate $\left[128^{*}, 131\right]$ and the kinetic energy of impinging droplets [132-134].
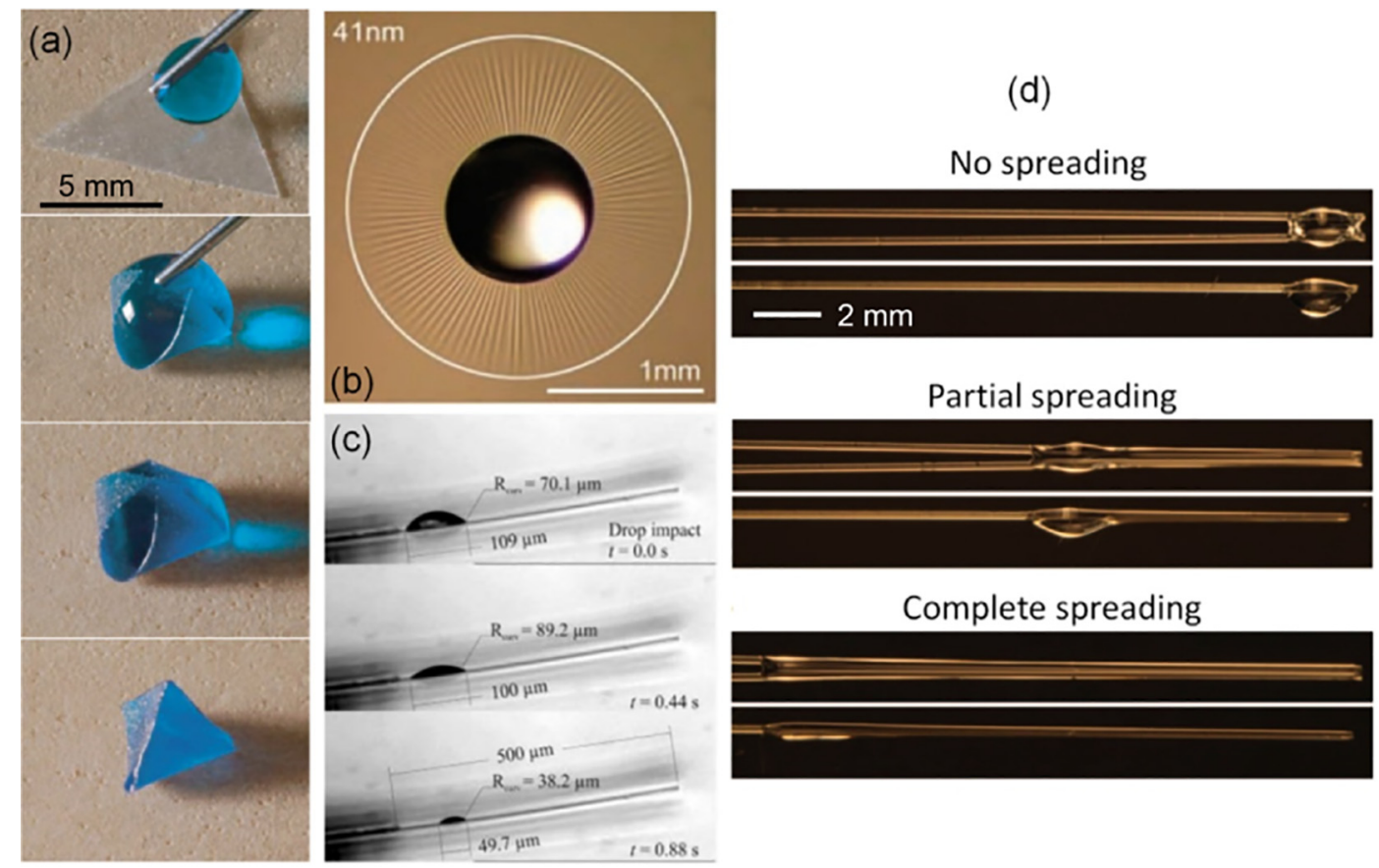

Fig. 7. (a) Wrapping of a water droplet with a triangular PDMS film via evaporation.

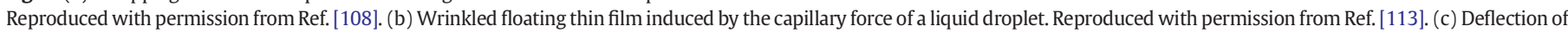

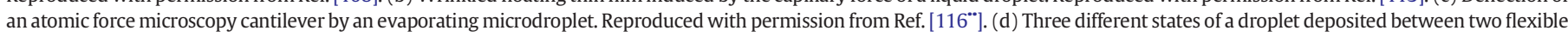
fibres. Reproduced with permission from Ref. [117]. 
Since the height of the wetting ridge primarily depends on the balance of the surface tensions, the thickness of the lubricating oil film does not affect the droplet sliding velocity [135]. Droplet impact experiments have shown that at low impact velocity (from 0.2 to $0.4 \mathrm{~m} / \mathrm{s}$ ) a thin air film can also form between the impinging droplet and the slippery substrate, similar to the soft viscoelastic substrates, and eventually the droplet rebounds from the substrate [133]. The rebound can be inhibited by increasing the free energy of the slippery surface via oxygen plasma treatment, and the impinging droplet undergoes a twostage spreading similar to that observed on rigid hydrophilic surfaces [136]. Droplet rebound was also observed at high impact velocity $(\gtrsim 1.5 \mathrm{~m} / \mathrm{s})$, when the kinetic energy of the impinging droplet is large enough to overcome the free energy of adhesion [137]. At very high impact velocity (up to $\sim 4.2 \mathrm{~m} / \mathrm{s}$ ), splashing was observed and the threshold velocity for splashing increases with the viscosity of the lubricant liquid [134], which resists the development of liquid instability.

The motion of the contact line during spreading and retraction for bouncing droplets on slippery substrates were found to be independent on viscosity of the infused oil [133]. However, the viscosity of the infused oil does influence the restitution coefficient of the bouncing droplets due to large viscous dissipation because of the substrate deformation near the contact line. It was also found that the spreading of non-bouncing droplets is substrate-independent, but the retraction stage is slowed down on infused oil with higher viscosity [133,134], and this behaviour is more significant as the viscosity ratio between the impinging droplet and infused oil layer increases [132]. The contact line dynamics of impacting droplets on slippery substrates has thus similarities with that on soft viscoelastic substrates $[16,71,90]$.

\section{Conclusions and outlook}

In the past years, the wetting of soft substrates has become a fastdeveloping area of research in soft condensed matter physics and interfacial science. Much theoretical, numerical and experimental work has been performed to understand the statics and dynamics of "soft" wetting.

Considering the static wetting problem, the substrate deformation due to capillary forces has been analysed using either a mechanical approach via a force balance or a thermodynamic approach via a minimization of the free energies. Although a good agreement between theoretical models and experimental results was reached for deformations away from the contact line, the region close to the contact line is still not finally resolved due to the strain singularity arising there. Moreover, additional effects such as the capillary force originating from the solid substrate and the Shuttleworth effect are not yet fully implemented in the theoretical analyses. While a rigorous theoretical model is still missing, numerical simulations based on continuum mechanics provide an alternative way to investigate substrate deformation profiles for soft solids over a wide range of stiffness. Spatial resolution is limited to the micrometre scale, though, and sub-micrometre effects are not captured by the simulations. From an experimental point of view, there has been fast progress in detecting first micrometre- and then nanometer-scale deformations between droplet and substrate by adapting imaging techniques like laser scanning confocal microscopy, environmental scanning electron microscopy, confocal fluorescence microscopy, or atomic force microscopy. The "apparent" contact angle of droplets on soft substrates has been found to depend on the size of the droplet, the elastocapillary length, the thickness of the soft substrate and the characteristic length of molecular interactions that determine the thickness of the prewetting liquid layer and the magnitude of the disjoining pressure. The elegant partial models developed that capture one or several of these effects call for experimental validation, especially for soft wetting at the micro- and nanoscale. One commonly agreed finding is that the wetting ridge acts as a mechanical defect on the surface and causes pinning of the contact line, with the consequence of increasing the contact angle hysteresis on soft substrates.
Considering the dynamic wetting problem, much efforts have been devoted to investigating the spreading of macroscopic droplets with a size (typically $\sim 1 \mathrm{~mm}$ ) much larger than the elastocapillary length. Four spreading regimes have been identified for the spontaneous wetting of soft substrates by low viscosity liquids. The spreading during the two "early" regimes behaves as that on very stiff substrates and the dynamics is dominated by inertial force. The two latter spreading regimes are dominated by the time and strain dependent viscoelastic properties of the soft substrate and by viscous dissipation within the droplet. In "soft" dynamic wetting systems, substrate deformation couples with capillary and hydrodynamic forces, causing rich and complex behaviours. Substrate deformation was also found to strongly affect droplet impact dynamics and to lead to new phenomena, such as droplet rebound or large bubble entrapment inside the droplet, in contrast to rigid substrates with similar surface properties. The contact line dynamics during drop impact and other wetting or dewetting processes could be understood by taking into consideration the rheological properties of the soft materials. A very basic approach for modelling wetting dynamics has been attempted by applying an energy balance to the spontaneous spreading of water droplets on viscoelastic substrates, employing linear viscoelasticity for modelling the mechanical response of the substrate strained by capillary and hydrodynamic forces [30-32*0]. Further progress in modelling dynamic wetting on soft substrates by capturing a larger number of physical effects is urgently needed, since most natural and industrial wetting processes on "soft" substrates are of dynamical nature. A comprehensive model relating capillary, hydrodynamic and mechanical/rheological aspects is the next important step to take.

Substrate stiffness has also been shown to play a key role in other capillary phenomena, such as droplet evaporation, dropwise condensation and frost growth. These aspects highlight the importance of designing further dedicated experimental investigations in "soft wetting" for collecting a larger database of representative effects and for supporting the development of more refined and possibly predictive models. This review article has been written with the intent to provide an overview of the most seminal and recent achievements and - as researchers interested in the further progress of "soft wetting" - we are looking forward to triggering the curiosity of even more investigators to dedicate their future thoughts and insights to this very interdisciplinary field.

\section{References and recommended reading,}

[1] de Gennes PG, Brochard-Wyart F, Quéré D. Capillarity and wetting phenomena. Springer; 2004.

[2] Starov VM, Velarde MG, Radke CJ. Wetting and spreading dynamics. New York, USA: CRC Press; 2007

[3] Zhao YP. Physical mechanics of surfaces and interfaces. Beijing: Science Press; 2012.

[4] Young T. An essay on the cohesion of fluids. Philos Trans R Soc Lond 1805;95: 65-87.

[5] Wenzel RN. Surface roughness and contact angle. J Phys Colloid Chem 1949;53: 1466-7.

[6] Cassie ABD, Baxter S. Wettability of porous surfaces. Trans Faraday Soc 1944;40: 546-51.

[7] Bico J, Thiele U, Quere D. Wetting of textured surfaces. Colloids Surf A Physicochem Eng Asp 2002;206:41-6.

[8] Bonn D, Eggers J, Indekeu J, Meunier J, Rolley E. Wetting and spreading. Rev Mod Phys 2009;81:739-805.

[9] de Gennes PG. Wetting: statics and dynamics. Rev Mod Phys 1985;57:827-63.

[10] Lester GR. Contact angles of liquids at deformable solid surfaces. J Colloid Sci 1961; 16:315-26.

[11] Shanahan MER, de Gennes PG. Adhesion 11. Netherlands: Springer; 1987

[12] Lester GR. Contact angles of liquids on organic solids. Nature 1966;209:1126-7. One of the first seminal papers discussing the topic of soft wetting and capturing some of the involved physics.

[13] Extrand CW, Kumagai Y. Contact angles and hysteresis on soft surfaces. J Colloid Interface Sci 1996;184:191-200.

[14] Carre A, Gastel JC, Shanahan MER. Viscoelastic effects in the spreading of liquids. Nature 1996;379:432-4. A more recent paper proposing a new model and showing the nanometer-size of a "dried" wetting ridge.

\footnotetext{
- Of special interest.

- Of outstanding interest.
} 
[15] Carre A, Shanahan MER. Direct evidence for viscosity-independent spreading on a soft solid. Langmuir 1995;11:24-6.

[16] Chen LQ Li ZG. Bouncing droplets on nonsuperhydrophobic surfaces. Phys Rev E 2010;82.

[17] Shanahan MER, Carre A. Viscoelastic dissipation in wetting and adhesion phenomena. Langmuir 1995;11:1396-402.

[18] Cao Z, Stevens MJ, Dobrynin AV. Adhesion and wetting of nanoparticles on soft surfaces. Macromolecules 2014;47:3203-9.

[19] Petit J, Bonaccurso E. General frost growth mechanism on solid substrates with different stiffness. Langmuir 2014;30:1160-8.

[20] Lopes MC, Bonaccurso E. Evaporation control of sessile water drops by soft viscoelastic surfaces. Soft Matter 2012;8:7875-81.

[21] Lopes MC, Bonaccurso E. Influence of substrate elasticity on particle deposition patterns from evaporating water-silica suspension droplets. Soft Matter 2013;9: 7942-50.

[22] Sokuler M, Auernhammer GK, Roth M, Liu CJ, Bonaccurso E, Butt HJ. The softer the better: fast condensation on soft surfaces. Langmuir 2010;26:1544-7.

[23] Rusanov AI. Theory of wetting of elastically deformed bodies.1. Deformation with a finite contact-angle. Colloid J USSR 1975;37:614-22. Seminal paper proposing an enriched physical model and discussing some of the fundamental problems of soft static wetting.

[24] Rusanov AI. Theory of wetting of elastically deformed bodies. 2. Equilibrium conditions and work of deformation with a finite contact angle. Colloid J USSR 1975;37: 623-8.

[25] Deryagin BV, Starov VM, Churaev NV. Pressure on a wetting perimeter. Colloid J USSR 1982:44:770-5.

[26] Shanahan MER, de Gennes PG. L'arête produite par un coin liquide près de la ligne triple de contact solide/liquide/fluide. C R Acad Paris 1986;2:517-21.

[27] Andreotti B, Bäumchen O, Boulogne F, Daniels KE, Dufresne ER, Perrin H, et al. Solid capillarity: when and how does surface tension deform soft solids? Soft Matter 2016;12:2993-6.

[28] Style RW, Jagota A, Hui CY, Dufresne ER. Elastocapillarity: surface tension and the mechanics of soft solids. In: Marchetti MC, Sachdev S, editors. Annu Rev. Condens Matter Phys. Palo Alto: Annual Reviews; 2017. p. 99-118.

[29] Pericet-Camara R, Auernhammer GK, Koynov K, Lorenzoni S, Raiteri R, Bonaccurso E. Solid-supported thin elastomer films deformed by microdrops. Soft Matter 2009; 5:3611-7.

[30] Shanahan MER. The spreading dynamics of a liquid drop on a viscoelastic solid. J Phys D Appl Phys 1988;21:981-5.

[31] Shanahan MER. The influence of solid micro-deformation on contact angle equilibrium. J Phys D Appl Phys 1987;20:945-50.

[32] Chen LQ, Bonaccurso E, Shanahan MER. Inertial to viscoelastic transition in early drop spreading on soft surfaces. Langmuir 2013;29:1893-8. First paper proposing a physical model based on an energy balance validated by experiments, and discussing some fundamental problems of soft dynamic wetting.

[33] White LR. The contact angle on an elastic substrate. 1 . The role of disjoining pressure in the surface mechanics. J Colloid Interface Sci 2003;258:82-96.

[34] Ahmed G, Kalinin VV, Arjmandi-Tash O, Starov VM. Equilibrium of droplets on a deformable substrate: influence of disjoining pressure. Colloids Surf A Physicochem Eng Asp 2017;521:3-12. The first paper where equilibrium contact angle was quantitatively deduced using disjoining pressure isotherm.

[35] Gelfand IM, Fomin SV. Calculus of variables. Englewood Cliffs, New Jersey: Prentice Hall, Inc; 1963.

[36] Koursary N, Ahmed G, Starov V. Conditions for equilibrium of liquid droplets on deformable substrates. Langmuir 2018 [submitted for publication].

[37] Long D, Ajdari A, Leibler L. Static and dynamic wetting properties of thin rubber films. Langmuir 1996;12:5221-30.

[38] Das S, Marchand A, Andreotti B, Snoeijer JH. Elastic deformation due to tangential capillary forces. Phys Fluids 2011;23.

[39] Marchand A, Das S, Snoeijer JH, Andreotti B. Capillary pressure and contact line force on a soft solid. Phys Rev Lett 2012;108.

[40] Jerison ER, Xu Y, Wilen LA, Dufresne ER. Deformation of an elastic substrate by a three-phase contact line. Phys Rev Lett 2011;106. Paper showing that also a tangential components pulls on the three-phase contact line and causes a substrate deformation.

[41] Style RW, Dufresne ER. Static wetting on deformable substrates, from liquids to soft solids. Soft Matter 2012;8:7177-84.

[42] Shuttleworth R. The surface tension of solids. Proc Phys Soc A 1950;63:444.

[43] Andreotti B, Snoeijer JH. Soft wetting and the Shuttleworth effect, at the crossroads between thermodynamics and mechanics. Europhys Lett 2016;113:66,001. Paper relating soft substrate deformation to a change of surface energy - and thus to wettability.

[44] Hui CY, Jagota A. Planar equilibrium shapes of a liquid drop on a membrane. Soft Matter 2015;11:8960-7.

[45] Schulman RD, Dalnoki-Veress K. Liquid droplets on a highly deformable membrane. Phys Rev Lett 2015;115:206,101.

[46] Neukirch S, Antkowiak A, Marigo JJ. Soft beams: when capillarity induces axial compression. Phys Rev E 2014;89:012401.

[47] Weijs JH, Andreotti B, Snoeijer JH. Elasto-capillarity at the nanoscale: on the coupling between elasticity and surface energy in soft solids. Soft Matter 2013;9: 8494-503.

[48] Madasu S, Cairncross RA. Static wetting on flexible substrates: a finite element formulation. Int J Numer Methods Fluids 2004;45:301-19.

[49] Yu YS, Yang ZY, Zhao YP. Role of vertical component of surface tension of the droplet on the elastic deformation of PDMS membrane. J Adhes Sci Technol 2008;22: 687-98.
[50] Yu YS, Zhao YP. Deformation of PDMS membrane and microcantilever by a water droplet: comparison between Mooney-Rivlin and linear elastic constitutive models. J Colloid Interface Sci 2009;332:467-76.

[51] Pericet-Camara R, Best A, Butt HJ, Bonaccurso E. Effect of capillary pressure and surface tension on the deformation of elastic surfaces by sessile liquid microdrops: an experimental investigation. Langmuir 2008;24:10565-8. First experimental paper based on laser scanning confocal microscopy recording complete 3D sub-micron deformations on soft substrates caused by microdrops; validation of Rusanov's White's models of "soft wetting".

[52] Gielok M, Lopes M, Bonaccurso E, Gambaryan-Roisman T. Droplet on an elastic substrate: finite element method coupled with lubrication approximation. Colloids Surf A Physicochem Eng Asp 2017;521:13-21.

[53] Leonforte F, Muller M. Statics of polymer droplets on deformable surfaces. J Chem Phys 2011;135.

[54] Heim LO, Bonaccurso E. Measurement of line tension on droplets in the submicrometer range. Langmuir 2013;29:14147-53.

[55] Berg JK, Weber CM, Riegler H. Impact of negative line tension on the shape of nanometer-size sessile droplets. Phys Rev Lett 2010;105.

[56] Andrade JD, King RN, Gregonis DE, Coleman DL. Surface characterization of poly (hydroxyethyl methacrylate) and related polymers. I. Contact angle methods in water. J Polym Sci Polym Symp 1979:313-36.

[57] Metois JJ. Elastic straining of a thin graphite layer by a liquid droplet or a nonepitaxed Pb crystallite. Surf Sci 1991;241:279-88.

[58] Saiz E, Tomsia AP, Cannon RM. Ridging effects on wetting and spreading of liquids on solids. Acta Mater 1998;46:2349-61.

[59] Pu G, Severtson SJ. Dependence of wetting behavior on the thickness of highly viscoelastic films. J Phys Chem C 2011;115:18729-35.

[60] Pu G, Guo JH, Gwin LE, Severtson SJ. Mechanical pinning of liquids through inelastic wetting ridge formation on thermally stripped acrylic polymers. Langmuir 2007; 23:12142-6.

[61] Pu G, Severtson SJ. Characterization of dynamic stick-and-break wetting behavior for various liquids on the surface of a highly viscoelastic polymer. Langmuir 2008;24:4685-92.

[62] Pu G, Ai J, Severtson SJ. Drop behavior on a thermally-stripped acrylic polymer: influence of surface tension induced wetting ridge formation on retention and running. Langmuir 2010;26:12696-702.

[63] Pu G, Severtson SJ. Water evaporation on highly viscoelastic polymer surfaces Langmuir 2012;28:10007-14.

[64] Style RW, Che Y, Wettlaufer JS, Wilen LA, Dufresne ER. Universal deformation of soft substrates near a contact line and the direct measurement of solid surface stresses. Phys Rev Lett 2013;110:066103. First paper trying - though in a simplified way - to relate contact angle and substrate deformations to the possibility of measuring the surface energy of soft solids.

[65] Park SJ, Weon BM, Lee JS, Lee J, Kim J, Je JH. Visualization of asymmetric wetting ridges on soft solids with X-ray microscopy. Nat Commun 2014;5:4369.

[66] Soon HY, Mu SJ. Contact angles on deformable solids. J Colloid Interface Sci 1985; $110: 252$.

[67] Marchand A, Das S, Snoeijer JH, Andreotti B. Contact angles on a soft solid: from Young's law to Neumann's law. Phys Rev Lett 2012;109. Paper trying to rationalize the wetting scenarios of sessile droplets on soft substrates caught in between Young's and Neumann's Laws.

[68] Chen LQ, Auernhammer GK, Bonaccurso E. Short time wetting dynamics on soft surfaces. Soft Matter 2011;7:9084-9.

[69] Lubbers LA, Weijs JH, Botto L, Das S, Andreotti B, Snoeijer JH. Drops on soft solids: free energy and double transition of contact angles. J Fluid Mech 2014;747:R1. Paper relating three length scales - size of the drop, molecular scale of the interface and elastocapillary length - with the macroscopic and microscopic equilibrium contact angles of drops on soft substrates. A further results was that total free energy is lower on softer substrates.

[70] Quere D. Wetting and roughness. Annu Rev. Mater Res. Palo Alto: Annual Reviews; 2008. p. 71-99.

[71] Chen LQ, Bonaccurso E, Deng PG, Zhang HB. Droplet impact on soft viscoelastic surfaces. Phys Rev E 2016;94:063117.

[72] Rioboo R, Voue M, Adao H, Conti J, Vaillant A, Seveno D, et al. Drop impact on soft surfaces: beyond the static contact angles. Langmuir 2010;26:4873-9.

[73] Lee JB, dos Santos S, Antonini C. Water touch-and-bounce from a soft viscoelastic substrate: wetting, dewetting, and rebound on bitumen. Langmuir 2016;32: 8245-54.

[74] Ahmed G, Koursari N, Kuchin I, Starov V. Hysteresis of contact angle of sessile droplets on deformable substrates: influence of disjoining pressure. Colloids Surf A Physicochem Eng Asp 2018 [submitted for publication]

[75] Kuchin I, Starov V. Hysteresis of contact angle of sessile droplets on smooth homogeneous solid substrates via disjoining/conjoining pressure. Langmuir 2015;31:5345-52. The first paper where static advancing/receding contact angles on non-deformable solid substrates were deduced via disjoining pressure isotherm.

[76] Winkels KG, Weijs JH, Eddi A, Snoeijer JH. Initial spreading of low-viscosity drops on partially wetting surfaces. Phys Rev E 2012;85.

[77] Bird JC, Mandre S, Stone HA. Short-time dynamics of partial wetting. Phys Rev Lett 2008;100.

[78] Chen LQ, Li CL, van der Vegt NFA, Auernhammer GK, Bonaccurso E. Initial electrospreading of aqueous electrolyte drops. Phys Rev Lett 2013;110.

[79] Courbin L, Bird JC, Reyssat M, Stone HA. Dynamics of wetting: from inertial spreading to viscous imbibition. J Phys Condens Matter 2009;21.

[80] Stapelbroek BBJ, Jansen HP, Kooij ES, Snoeijer JH, Eddi A. Universal spreading of water drops on complex surfaces. Soft Matter 2014;10:2641-8. 
[81] Shanahan MER, Carre A. Anomalous spreading of liquid drops on an elastomeric surface. Langmuir 1994;10:1647-9.

[82] Grant CA, Twigg PC, Savage MD, Woon WH, Wilson M, Greig D. Estimating the mechanical properties of retinal tissue using contact angle measurements of a spreading droplet. Langmuir 2013;29:5080-4.

[83] Nonomura Y, Morita Y, Hikima T, Seino E, Chida S, Mayama H. Spreading behavior of water droplets on fractal agar gel surfaces. Langmuir 2010;26:16150-4.

[84] Nonomura Y, Chida S, Seino E, Mayama H. Anomalous spreading with Marangoni flow on agar gel surfaces. Langmuir 2012;28:3799-806.

[85] Banaha M, Daerr A, Limat L. Spreading of liquid drops on Agar gels. Eur Phys J Spec Top 2009; 166:185-8.

[86] Kajiya T, Daerr A, Narita T, Royon L, Lequeux F, Limat L. Dynamics of the contact line in wetting and diffusing processes of water droplets on hydrogel (PAMPS-PAAM) substrates. Soft Matter 2011;7:11425.

[87] Saiz E, Cannon RM, Tomsia AP. High-temperature wetting and the work of adhesion in metal/oxide systems. Annu Rev. Mater Res. Palo Alto: Annual Reviews; 2008. p. 197-226.

[88] Eustathopoulos N. Progress in understanding and modeling reactive wetting of metals on ceramics. Curr Opin Solid State Mater Sci 2005;9:152-60.

[89] Saiz E, Tomsia AP. Kinetics of high-temperature spreading. Curr Opin Solid State Mater Sci 2005;9:167-73.

[90] Chen LQ Wu J, Li ZG, Yao SH. Evolution of entrapped air under bouncing droplets on viscoelastic surfaces. Colloids Surf A Physicochem Eng Asp 2011;384:726-32.

[91] Renardy Y, Popinet S, Duchemin L, Renardy M, Zaleski S, Josserand C, et al. Pyramidal and toroidal water drops after impact on a solid surface. J Fluid Mech 2003;484 69-83.

[92] Howland CJ, Antkowiak A, Castrejon-Pita JR, Howison SD, Oliver JM, Style RW, et al. It's harder to splash on soft solids. Phys Rev Lett 2016;117:184502. Paper showing the suppression of drop splashing by tuning substrate stiffness.

[93] Mangili S, Antonini C, Marengo M, Amirfazli A. Understanding the drop impact phenomenon on soft PDMS substrates. Soft Matter 2012;8:10045-54.

[94] Alizadeh A, Bahadur V, Shang W, Zhu Y, Buckley D, Dhinojwala A, et al. Influence of substrate elasticity on droplet impact dynamics. Langmuir 2013;29:4520-4.

[95] Pu G, Severtson SJ. Variety of wetting line propagations demonstrated on viscoelastic substrate. Appl Phys Lett 2009;94.

[96] Kajiya T, Daerr A, Narita T, Royon L, Lequeux F, Limat L. Advancing liquid contact line on visco-elastic gel substrates: stick-slip vs. continuous motions. Soft Matter 2013;9:454-61.

[97] Kajiya T, Brunet P, Royon L, Daerr A, Receveur M, Limat L. A liquid contact line receding on a soft gel surface: dip-coating geometry investigation. Soft Matter 2014; 10:8888-95.

[98] Kajiya T, Brunet P, Daerr A, Royon L, Narita T, Lequeux Fo, et al. Wetting on gels: how the gel characteristics affect the contact line dynamics? Interfacial Phenom Heat Transf 2013;1:215-30.

[99] Yu YS, Wang ZQ Zhao YP. Experimental study of evaporation of sessile water droplet on PDMS surfaces. Acta Mech Sinica 2013;29:799-805.

[100] Bonaccurso E, Butt H-J, Hankeln B, Niesenhaus B, Graf K. Fabrication of microvessels and microlenses from polymers by solvent droplets. Appl Phys Lett 2005;86: 124102.

[101] Pericet-Camara R, Nett S, Best A, Gutmann J, Bonaccurso E. Arrays of microlenses with variable focal lengths fabricated by restructuring polymer surfaces with an ink-jet device. Opt Express 2007;15:9877-82.

[102] Eslami F, Elliott JA. Thermodynamic investigation of the barrier for heterogeneous nucleation on a fluid surface in comparison with a rigid surface. J Phys Chem B 2011;115:10646-53.

[103] Style RW, Che YL, Park SJ, Weon BM, Je JH, Hyland C, et al. Patterning droplets with durotaxis. Proc Natl Acad Sci U S A 2013;110:12541-4.

[10.4] Phadnis A, Rykaczewski K. Dropwise condensation on soft hydrophobic coatings. Langmuir 2017;33:12095-101. A more recent paper demonstrating the reduction of condensation heat transfer by softening the solid substrate.

[105] Guo XY, Li H, Ahn BY, Duoss EB, Hsia KJ, Lewis JA, et al. Two- and three-dimensiona folding of thin film single-crystalline silicon for photovoltaic power applications. Proc Natl Acad Sci U S A 2009;106:20149-54.

[106] Py C, Reverdy P, Doppler L, Bico J, Roman B, Baroud CN. Capillary origami: spontaneous wrapping of a droplet with an elastic sheet. Phys Rev Lett 2007;98.

[107] Chen LQ, Wang X, Wen WJ, Li ZG. Critical droplet volume for spontaneous capillary wrapping. Appl Phys Lett 2010;97.

[108] Py C, Reverdy P, Doppler L, Bico J, Roman B, Baroud CN. Capillarity induced folding of elastic sheets. Eur Phys J Spec Top 2009;166:67-71.

[109] Wang ZQ, Wang FC, Zhao YP. Tap dance of a water droplet. Proc R Soc A 2012;468: 2485-95.

[110] Yuan QZ, Zhao YP. Precursor film in dynamic wetting, electrowetting, and electroelasto-capillarity. Phys Rev Lett 2010;104.
[111] Zhu XY, Yuan QZ, Zhao YP. Capillary wave propagation during the delamination of graphene by the precursor films in electro-elasto-capillarity. Sci Rep 2012;2.

[112] Nadermann N, Hui CY, Jagota A. Solid surface tension measured by a liquid drop under a solid film. Proc Natl Acad Sci U S A 2013;110:10541-5.

[113] Huang J, Juszkiewicz M, de Jeu WH, Cerda E, Emrick T, Menon N, et al. Capillary wrinkling of floating thin polymer films. Science 2007;317:650-3.

[114] Schroll RD, Adda-Bedia M, Cerda E, Huang J, Menon N, Russell TP, et al. Capillary deformations of bendable films. Phys Rev Lett 2013;111:014301.

[115] Toga KB, Huang J, Cunningham K, Russell TP, Menon N. A drop on a floating sheet: boundary conditions, topography and formation of wrinkles. Soft Matter 2013;9: 8289-96.

[116] Bonaccurso E, Butt H-J. Microdrops on atomic force microscope cantilevers: evaporation of water and spring constant calibration. J Phys Chem B 2004;109:253-63. First paper visualizing in real-time and modelling the combined effects of capillary pressure, non-balanced surface tensions, and line tension during the evaporation of microdrops on thin elastic Atomic Force Microscope cantilevers.

[117] Duprat C, Protiere S, Beebe AY, Stone HA. Wetting of flexible fibre arrays. Nature 2012;482:510-3. Paper analysing liquid drops deposited on pairs of flexible fibres and showing that flexibility, fibre geometry and drop volume are the relevant parameters that allow predicting when fibres coalesce or not.

[118] Bonaccurso E, Gillies G. Revealing contamination on AFM cantilevers by microdrops and microbubbles. Langmuir 2004:20:11824-7.

[119] Bonaccurso E. Microdrops evaporating on AFM cantilevers. Progr Colloid Polym Sci 2008;134:57-65.

[120] Golovko DS, Bonanno P, Lorenzoni S, Stefani F, Raiteri R, Bonaccurso E. Evaporative cooling of sessile water microdrops measured with atomic force microscope cantilevers. J Micromech Microeng 2008;18:095026.

[121] Haschke T, Bonaccurso E, Butt H-J, Lautenschlager D, Schönfeld F, Wiechert W. Sessile-drop induced bending of atomic force microscope cantilevers: a model system for monitoring microdrop evaporation. J Micromech Microeng 2006:2273-80.

[122] Liu C, Liu Y, Sokuler M, Fell D, Keller S, Boisen A, et al. Diffusion of water into SU-8 microcantilevers. Phys Chem Chem Phys 2010;12:10577-83.

[123] Liu C, Lopes MC, Pihan SA, Fell D, Sokuler M, Butt H-J, et al. Water diffusion in polymer nano-films measured with microcantilevers. Sensors Actuators B 2011;160: 32-8.

[124] Chen LQ Heim L-O, Golovko DS, Bonaccurso E. Snap-in dynamics of single particles to water drops. Appl Phys Lett 2012;101:031601.

[125] Duprat C, Bick AD, Warren PB, Stone HA. Evaporation of drops on two parallel fibers: influence of the liquid morphology and fiber elasticity. Langmuir 2013;29: 7857-63.

[126] Wong TS, Kang SH, Tang SKY, Smythe EJ, Hatton BD, Grinthal A, et al. Bioinspired self-repairing slippery surfaces with pressure-stable omniphobicity. Nature 2011; 477:443-7.

[127] Lafuma A, Quéré D. Slippery pre-suffused surfaces. Europhys Lett 2011;96:56001.

[128] Smith JD, Dhiman R, Anand S, Reza-Garduno E, Cohen RE, McKinley GH, et al. Droplet mobility on lubricant-impregnated surfaces. Soft Matter 2013;9:1772-80. Paper analyzing the thermodynamic configurations and mobility of droplets placed on liquid-infused substrates.

[129] Anand S, Paxson AT, Dhiman R, Smith JD, Varanasi KK. Enhanced condensation on lubricant-impregnated nanotextured surfaces. ACS Nano 2012;6:10122-9.

[130] Anand S, Rykaczewski K, Subramanyam SB, Beysens D, Varanasi KK. How droplets nucleate and grow on liquids and liquid impregnated surfaces. Soft Matter 2015; 11:69-80.

[131] Daniel D, Mankin MN, Belisle RA, Wong T-S, Aizenberg J. Lubricant-infused micro/ nano-structured surfaces with tunable dynamic omniphobicity at high temperatures. Appl Phys Lett 2013;102:231603.

[132] Kim JH, Rothstein JP. Droplet impact dynamics on lubricant-infused superhydrophobic surfaces: the role of viscosity ratio. Langmuir 2016;32: 10166-76.

[133] Hao C, Li J, Liu Y, Zhou X, Liu Y, Liu R, et al. Superhydrophobic-like tunable droplet bouncing on slippery liquid interfaces. Nat Commun 2015;6:7986.

[134] Lee C, Kim H, Nam Y. Drop impact dynamics on oil-infused nanostructured surfaces. Langmuir 2014;30:8400-7.

[135] Eifert A, Paulssen D, Varanakkottu SN, Baier T, Hardt S. Simple fabrication of robust water-repellent surfaces with low contact-angle hysteresis based on impregnation. Adv Mater Interfaces 2014;1:1300138.

[136] Multanen V, Whyman G, Shulzinger E, Valtsifer V, Bormashenko E. Plasma treatment of silicone oil- infused surfaces switches impact of water droplets from bouncing to tanner-like spreading. Colloids Surf A Physicochem Eng Asp 2018; 538:133-9.

[137] Chen LQ, Geissler A, Bonaccurso E, Zhang K. Transparent slippery surfaces made with sustainable porous cellulose lauroyl ester films. ACS Appl Mater Interfaces 2014;6:6969-76. 